\title{
Historia Social de la Ciencia: "Moluscos de la Isla del Coco" (1935) de Paul Biolley
}

\section{Ronald Eduardo Díaz-Bolaños ${ }^{1,2,3}$}

1. Centro de Investigaciones Geofísicas, Ciudad de la Investigación, Universidad de Costa Rica, San Pedro, 11501-2060 San José, Costa Rica, roeddibo@yahoo.com.

2. Escuela de Estudios Generales, Universidad de Costa Rica.

3. Cátedra de Historia, Escuela de Ciencias Sociales y Humanidades, Universidad Estatal a Distancia, San Pedro, 4742050 San José, Costa Rica.

Recibido 30-I-2019. Corregido 15-V-2019. Aceptado 25-VI-2019.

\begin{abstract}
Analysis of the paper: "Mollusks of Cocos Island" (1935) by Paul Biolley from the Social History of Science. Introduction: The paper "Moluscos de la Isla del Coco" (Mollusks of Cocos Island) published in the Revista del Colegio Superior de Señoritas (1935) is the translation to Spanish of one of the last works of Paul Biolley Matthey (1862-1908), Swiss naturalist that lived in Costa Rica. This article was one product of a malacological research carried out during a scientific expedition organized by the 'Instituto FísicoGeográfico Nacional de Costa Rica' (IFGN) in 1902 to Cocos Island, at a time when the country claimed its sovereignty both in this territory. Objective: To analyze the contributions of the article "Moluscos de la Isla del Coco" from the Social History of Science approach. Methods: Most of the researches that have applied this approach in Costa Rica analyze processes of institutionalization of scientific disciplines. The methodology used in these works was very useful for the analysis of the Biolley article, together with the information of historical data provided by other texts about Cocos Island and the development of scientific research in Costa Rica from a temporal perspective. In addition, tables were made to synthesize the classification of the species and genera studied by Biolley in Cocos Island to identify their provenance and the habitat in which they lived. Results: This study of Biolley constitutes a text of interest from the point of view of the Social History of Science, due to the descriptions it offers of the physical conditions of the territory of Cocos Island, of the different species of mollusks located in them and to the exchange of information with national and foreign scientists that show the participation of Biolley in scientific networks. Conclusions: The analysis of the information with the Social History of Science approach, concludes that the compilation of the flora and fauna permits a greater knowledge of the natural resources of the explored territories, in order to colonize them and integrate them into the economic dynamics of the Central Valley of Costa Rica. However, attempts colonize Cocos Island failed given the distance between this and the continent, in addition to the topographic conditions that prevent the permanent establishment of human populations and their productive activities.
\end{abstract}

Key words: Costa Rica, Cocos Island, History, Geography, Science, Mollusks.

Díaz-Bolaños, R. E. (2020). Historia Social de la Ciencia: "Moluscos de la Isla del Coco" (1935) de Paul Biolley. Revista de Biología Tropical, 68(Supl. 1), S296-S305.

La Isla del Coco, situada a $5^{\circ} 32^{\prime} \mathrm{N}$ y $87^{\circ} 04^{\prime} \mathrm{W}$, es un territorio insular costarricense en el Pacífico Tropical Oriental y, con sus $24 \mathrm{~km}^{2}$ terrestres, constituye el único afloramiento de la Cordillera Volcánica del Coco que sobresale de la placa homónima (Díaz,
Alfaro \& Ugalde, 2012). Esta isla, que durante el período colonial fue originalmente refugio de piratas y balleneros, fue objeto de varias expediciones con objetivos científicos desde el siglo XVIII hasta el presente y de acuerdo con Cortés (2008), entre las exploraciones que 
se han documentado destacan las de Malaspina (1791), Colnett (1793), Vancouver (1795), Belcher (1836), De Gueydon (1846), Agassiz (1888, 1891), Lièvre (1889), McCartney Passmore (1895), Hopkins (1898-1899) y Pittier (1898, 1902).

La expedición de 1902 fue organizada por el Instituto Físico-Geográfico Nacional (IFGN), en ese momento era una de las principales instituciones científicas con que contaba Costa Rica, cuyo responsable fue el naturalista suizo Henri Pittier Dormond (1857-1950), director de la institución desde su fundación en 1889 y hasta su renuncia en 1904. Esta institución, junto con el Museo Nacional de Costa Rica, fueron parte de una serie de entidades científicas pertenecientes a lo que Viales \& Clare (2009) denominaron "régimen de cientificidad", concepto que designa un conjunto de factores políticos, económicos, sociales, culturales y científicos que se amalgamaron para propiciar el desarrollo de la actividad científica en la Costa Rica de finales del siglo XIX e inicios del siglo XX. Esta propuesta se contrapone a la tesis del "aislacionismo científico", propuesta por Coronado (1997), según la cual, la actividad científica decayó en el país tras la renuncia de Pittier de la dirección del IFGN y la fundación de la Universidad de Costa Rica en 1940.

El profesor y naturalista suizo Paul Biolley Matthey (1862-1908) era parte del personal que participó en dicha expedición, que tuvo lugar en el mes de enero de 1902. Biolley, había arribado a Costa Rica en 1886 como miembro del cuerpo docente contratado en Europa para asumir el profesorado en las instituciones de enseñanza surgidas en el marco de la reforma educativa impulsada por el gobierno liberal del Gral. Bernardo Soto (1885-1889), por lo que se dedicó a impartir lecciones de Ciencias Físicas y Naturales en el Liceo de Cosa Rica, el Colegio Superior de Señoritas y el Colegio San Luis Gonzaga (Díaz \& Solano, 2008).

Paul Biolley había ejercido la dirección interina del IFGN en 1889 y 1903, en ausencia de Henri Pittier y a lo largo de su vida publicó varias obras relacionadas con la geografía y la historia natural de Costa Rica, entre ellas Elementos de historia natural (1887), Costa Rica et son avenir (1889), Invertebrados de Costa Rica (1895-1897), Moluscos terrestres y fluviátiles de la Meseta Central de Costa Rica (1897) y Ortópteros recogidos en Costa Rica desde 1890 á 1900 (1900) (Díaz \& Solano, 2009).

Esta expedición coincidió con los últimos meses de la administración del presidente Rafael Iglesias Castro (1894-1902), bajo cuyo mandato el IFGN había realizado otra expedición similar en 1898 y en ambos casos, la organización de viajes de exploración científica tenía como fin la reafirmación de la soberanía de Costa Rica sobre la Isla del Coco, que ya contaba con un gobernador, una presencia militar y el constante arribo de buques mercantes con bandera nacional (Arias, 1993). El naturalista suizo Henri Pittier lideró este viaje de investigación científica en el que se investigaron las condiciones geográficas y la biodiversidad de la Isla del Coco, en la que Paul Biolley cumplió con la función de hacer un inventario de los moluscos localizados y que sería publicado hasta 1907 (Cortés, 2008).

\section{METODOLOGÍA Y FUENTES DOCUMENTALES}

La fuente principal para la elaboración de la presente investigación fue la versión en español del texto Mollusques de l'isla del Coco publicada como artículo en 1935 y conservado en los acervos de la Biblioteca Nacional Miguel Obregón Lizano de Costa Rica. El texto original fue escrito en francés, lengua materna de Biolley, bajo el título Mollusques de l'isla del Coco: Résultats d'une expédition faîte en janvier 1902- du 11 au 16- sous les auspices du Gouvernement de Costa Rica y publicado en formato de fascículo por el Museo Nacional de Costa Rica en 1907 (Biolley, 1907). A este le siguió una traducción parcial que fue incluida en los Anales del Museo Nacional de El Salvador (1908-1909) a manera de homenaje póstumo para su autor (Díaz \& Solano, 2008). Finalmente, la traducción completa fue publicada en la Revista del Colegio Superior 
de Señoritas (1935) y realizada por Claudia Martínez Nussbaumer (1902-1992), quien se desempeñó como profesora de lengua francesa en dicha institución educativa, de la que el propio Biolley fue también docente.

El artículo original fue publicado gracias a las gestiones efectuadas por el naturalista costarricense Anastasio Alfaro González (18651951), como director del IFGN (1904-1924), entidad que se había incorporado institucionalmente al Museo Nacional, que Alfaro dirigía desde su fundación en 1887. Los mapas que fueron impresos en el texto original fueron obra del científico costarricense José Fidel Tristán Fernández (1873-1932), que se había destacado por sus investigaciones en el campo de las ciencias naturales y fue un activo colaborador del naturalista suizo radicado en Costa Rica (Biolley, 1935; Díaz, 2003).

Este artículo se compone de las siguientes secciones: 1) Introducción, una amplia sección en el que se describe las condiciones físicas de la Isla del Coco, 2) Bibliografía, en el que enumera las principales obras citadas en su artículo y 3) Enumeración de las especies, en el que describe cada una de las especies investigadas. Además, el autor añadió varias notas para ampliar los contenidos de su obra, confrontar las opiniones de otros investigadores o incorporar información adicional para esclarecer algunos aspectos de la investigación.

La obra fue analizada mediante una revisión del texto en el que se sintetizaron las principales características físicas de la Isla del Coco, se confeccionaron tablas con los nombres de las especies y los géneros basados en los criterios empleados por Biolley para clasificar los moluscos que estudió en la isla. Además, se extrajo información de la tesis de Arias (1993) sobre la historia de la Isla del Coco, para el análisis del contexto histórico en que tuvo lugar la expedición de 1902 y se empleó la metodología propuesta por Solano (1999), Díaz (2003) y Solano, Díaz \& Amador (2010 y 2013) para analizar textos empleando el enfoque de Historia Social de la Ciencia, que a su vez fue planteada por
Saldaña (1996) para estudiar la experiencia histórico-científica latinoamericana.

\section{RESULTADOS}

Biolley (1935) realiza una descripción básica de la Isla del Coco al caracterizar su ubicación geográfica a partir de sus coordenadas y al punto más próximo con respecto al territorio continental costarricense, que el autor debate si era el Cabo Salsipuedes en la Península de Osa o bien el Cabo Blanco en la Península de Nicoya. Calcula la superficie de la isla en 46,6 $\mathrm{km}^{2}$, casi el doble de su verdadera extensión y la altura máxima en $675 \mathrm{msnm}$, la que llevaría el nombre de Cerro Iglesias en honor al presidente Rafael Iglesias Castro (1861-1924). Describe las costas y los islotes habitados por aves marinas que rodean la isla, advierte de sus irregularidades y las condiciones favorables para el fondeadero de embarcaciones en las bahías Chatham y Wafer, además de la abrupta topografía del interior y los escasos cursos hidrográficos que riegan su territorio que son alimentados por las constantes precipitaciones anuales y a su vez favorecen una temperatura agradable. Dada su distancia del continente y las condiciones físicas de la misma, los proyectos de convertirla en un centro penal o una colonia agrícola habían fracasado, pero se auguraba la transformación en una estación para el abastecimiento de carbón con la futura apertura del Canal de Panamá.

Con base en las hipótesis planteadas por Geor Baur (1859-1898) y Henri MilneEdwards (1800-1885) sobre el origen geológico de las vecinas Galápagos, sostiene el origen volcánico de la isla, pero con una conformación distinta a la del archipiélago ecuatoriano. Biolley encuentra similitudes entre la escasa fauna isleña y la que se ubica en el continente entre México y Perú, pero dado el estado de las investigaciones zoológicas efectuadas en la isla, no profundiza en dicha temática, con el fin de efectuar un análisis más comparativo entre sus hallazgos y los de los autores que le precedieron. 
En cuanto a la vegetación, confronta la tesis de Henri Pittier, que propone un carácter autóctono frente a la de Alexander Agassiz (1835-1910), quien sostuvo más bien que fue introducida, gracias a las corrientes marinas. Este último punto fue cuestionado por los autores Robert Evans Snodgrass (1875-1962) y Edmund Heller (1875-1939), quienes consideran que el transporte de animales y plantes hacia la Isla del Coco era improbable dado los cursos que toman las corrientes marinas en esa zona del Océano Pacífico. Biolley describe la presencia de una flora exuberante, en la que destacan los helechos en el interior y las Ipomea cerca de la costa, además de las palmeras, los guarumos y los árboles de balsa (Ochroma lagopus $S w$.), aunque no profundiza en aspectos relacionados con la botánica.

Biolley (1935) agrega a manera de nota, respecto a la polémica generada por el papel de las corrientes marinas en la colonización de la flora y fauna insular, la experiencia realizada por Anastasio Alfaro, quien arrojó una botella con un mensaje en un punto del océano, aproximadamente a media distancia entre Puntarenas y la Isla del Coco, el 12 de junio de 1898. Dicha botella fue recogida por el Reverendo C. F. Rife en la isla Ailuk (Islas Marshall) el 15 de octubre de 1906, quien comunicó el hallazgo a la Oficina Hidrográfica de los Estados Unidos, que a su vez informó a Alfaro, entonces director del Instituto Físico-Geográfico Nacional, del suceso. En una carta posterior, se detalla el posible rumbo de la mencionada botella, que las corrientes marinas pudieron transportarla frente a las costas centroamericana, mexicana y californiana hasta adentrarse en el océano y posteriormente enrumbarse hacia la Micronesia donde se produjo el hallazgo.

El naturalista mantuvo intercambio científico con el malacólogo francés César Marie Félix Ancey (1860-1906), con el fin de analizar los moluscos localizados en la Isla del Coco y debatir sobre los estudios malacológicos efectuados en ese territorio insular desde inicios del siglo XIX. Al comparar sus estudios con los de Ancey, el naturalista radicado en Costa Rica descarta su opinión indicando que a pesar del tamaño de la isla, existe una diversidad de moluscos (en particular los terrestres) y no unos cuantos como opinaba su par francés. Además, se apoyó también en el estudio efectuado por Henry Augustus Pilsbry (1862-1957) y E. G. Vanatta sobre los moluscos marinos de las islas Galápagos, para compararlos con sus resultados de investigación obtenidos en la Isla del Coco (Biolley, 1935). El autor del artículo efectuó una clasificación de los moluscos analizados en sus investigaciones y en las existentes y los distribuyó como se muestra en la Tabla 1 y la Tabla 2.

El método empleado por Biolley fue el de recolectar los especímenes de moluscos in situ para luego describir sus características, ubicando el sitio donde fueron localizados con el fin de identificar su hábitat. Sus resultados eran comparados con los proporcionados por estudios anteriores, por lo que recurre a una abundante producción bibliográfica elaborada entre el siglo XIX y los primeros años del siglo $\mathrm{XX}$, que le permite ubicarlos también en diferentes puntos de la costa pacífica del continente americano y de las islas de Oceanía e incluso de otras regiones tropicales del planeta.

Biolley indicó otros aportes, en notas al pie de página, efectuados durante sus trabajos de investigación en la Isla del Coco, como lo fueron el hallazgo del Anolis townsendi Stenj. y del Sphaerodactylus pacifus, reptiles que fueron descritos por el zoólogo noruego Leonhard Stejneger (1851-1943) en el primer lustro del siglo XX y destacó la presencia del miriápodo

TABLA 1

Género o especies de moluscos según su procedencia geográfica. Fuente: Biolley (1935)

\begin{tabular}{|c|c|}
\hline Procedencia & Género o especie \\
\hline Propia & $\begin{array}{l}\text { Guppya } \\
\text { Succinea } \\
\text { Vertigo }\end{array}$ \\
\hline América & $\begin{array}{l}\text { Auricula } \\
\text { Neritina }\end{array}$ \\
\hline Polinesia & $\begin{array}{l}\text { Acmaea striata Q. G. } \\
\text { Tornatellina } \\
\text { Opeas junceum Gould (¿cosmopolita?) }\end{array}$ \\
\hline Introducida & Leptinaria biolleyi v. Mart. \\
\hline
\end{tabular}


TABLA 2

Clasificación según el tipo de hábitat de los moluscos. Fuente: Biolley (1935)

\begin{tabular}{|c|c|}
\hline Hábitat & Especie \\
\hline Terrestre & $\begin{array}{l}\text { Guppya pacifica } \text { Pfr. } \\
\text { Guppya fultoni Gude } \\
\text { Tornatellina pittieri v. Mart. } \\
\text { Leptinarias biolleyi v. Mart. } \\
\text { Opeas junceum H. Gould } \\
\text { Vertigo cococensis Dall } \\
\text { Succinea } \text { sp. } \\
\text { Succinea globispira v. Mart. }\end{array}$ \\
\hline Agua dulce o salobre & $\begin{array}{l}\text { Neritina latissina Brod. } \\
\text { Auricula (?) stagnalis Orb. }\end{array}$ \\
\hline Marino & $\begin{array}{l}\text { Conus brunneus Wood } \\
\text { Purpura patula } \text { L. } \\
\text { Purpura columellaris Lam. } \\
\text { Purpura melones Duclos } \\
\text { Monoceros brevidentatum Gray } \\
\text { Pollia sanguinolenta Duclos (1932)/Pollia haemostoma Gray } \\
\text { Pollia cinis Reeve } \\
\text { Ranella caelata } \text { Brod. } \\
\text { Cypraea isabella } \text { L. (?) } \\
\text { Cerithium adustum, Kien } \\
\text { Planaxis planicostatus Sow } \\
\text { Littorina aspera } \text { Phill. } \\
\text { Hyponnix grayanus } \text { Mke. } \\
\text { Nerita ornata } \text { Sow } \\
\text { Nerita bernhardi } \text { Recluz } \\
\text { Fissurella virescens } \text { Sow } \\
\text { Acmaea striatata } \text { Q. G. } \\
\text { Chiton } \text { (Radsia) } \text { stokesi Brod. } \\
\text { Melampus tabogensis } \text { C.B. Adams } \\
\text { Siphonaria gigas Sow } \\
\text { Ostraea ochracea } \text { Sow } \\
\text { Perna quadrangularis Reeve } \\
\text { Vermetus } \text { sp. }\end{array}$ \\
\hline
\end{tabular}

Rhinocricus (Eurhinocricus) biolleyi, como un ejemplo de un invertebrado que es posible localizar tanto en la isla como en el territorio continental costarricense. Además, varios especímenes de invertebrados procedentes de esta isla, colectados por Biolley, fueron estudiados por diversos investigadores como el miriapodólogo francés Henry Brolemann (1860-1933), el aracnólogo estadounidense Nathan Banks (1868-1953), el mirmecólogo suizo Auguste Forel (1848-1931), el entomólogo jesuita austríaco Erich Wasmann (1869-1931) y el carcinólogo inglés y reverendo anglicano Thomas R. R. Stebbing (1835-1926) (Biolley, 1935). Este intercambio evidencia la participación de Biolley en una red científica internacional, gracias al envío de especímenes de invertebrados que eran investigados por científicos de varios países.

Finalmente, mencionó las contribuciones realizadas por el naturalista inglés Hugh Cuming (1791-1865), quien pudo haber sido el primer investigador en colectar conchas en la Isla del Coco hacia 1827, además de los aportes hechos por el malacólogo alemán Eduard von Martens (1831-1904), el británico Gerard Pierre Laurent Kalshoven Gude (18581924) y el estadounidense William Healey Dall (1845-1927), en el estudio de los moluscos de la Isla del Coco, además de las investigaciones 
hechas por el naturalista costarricense Anastasio Alfaro en el campo de la mastozoología y por Charles Haskins Townsend (1859-1944), Robert Evans Snodgrass y Edmund Heller en el de la ornitología.

De esta forma, los especímenes recolectados por Biolley, tanto de moluscos como de otras especies de la fauna presentes en la Isla del Coco que fueron enviados a científicos radicados en Washington D.C. (Estados Unidos) y en varios países europeos, principalmente en Alemania y Francia, fueron incorporados a las colecciones organizadas por estos investigadores que junto con otras muestras procedentes de otros puntos del planeta, pudieron ser posteriormente incorporadas a museos y otras instituciones científicas. Esto fue el inicio de las primeras colecciones malacológicas relacionadas con moluscos recolectados en Costa Rica y gracias a ellas fue posible obtener información de los moluscos presentes en dicho territorio insular costarricense.

\section{DISCUSIÓN}

El análisis de la versión en español del artículo de Biolley constituye una contribución al estudio de los moluscos presentes en la Isla del Coco. La versión original en francés fue citada por Monje-Nájera (1997) en su estudio sobre los moluscos de Costa Rica y por Barrientos (2003) en su investigación sobre los moluscos terrestres localizados en el mismo país centroamericano. Monje-Nájera (1997) incluye una reseña histórica de la malacología centroamericana en el que menciona el aporte de los estudios de Biolley en dicho campo, no obstante, no se refiere al contexto histórico en el que se produjeron.

Desde el punto de vista de la Historia Social de la Ciencia, este trabajo constituye, además de la descripción de las condiciones físicas del territorio insular costarricense, un estudio bastante pormenorizado de las distintas especies de moluscos que el naturalista suizo pudo recolectar en la expedición de 1902. Esta relación pormenorizada es característica de las expediciones organizadas por el IFGN en el que era importante recopilar las especies de flora y fauna de los territorios explorados, al mismo tiempo que se recababan datos geográficos para incorporarlos en el Mapa de Costa Rica que fue publicado en 1903; esto con la finalidad de identificar los recursos potenciales con que las zonas fronterizas y costeras contaban, para que dichos espacios fueran colonizados en el futuro e integrarlos en la economía del Valle Central de Costa Rica, basada primordialmente en la producción cafetalera (Díaz, 2007, 2009). Esa idea de investigar la naturaleza, considerada prístina en un sitio o región escasamente habitado o intervenido por los seres humanos, particularmente en las regiones tropicales del planeta, con la finalidad de conocer su potencial económico para luego explotarlo correspondía a una visión utilitarista, ligada a la lógica del sistema capitalista, que fue compartida por los científicos, exploradores, misioneros y viajeros europeos y estadounidenses que visitaron Costa Rica entre mediados del siglo XIX y la primera mitad del siglo XX (Quesada, 2001; Goebel, 2009).

Es evidente también que estas expediciones tenían como fin reafirmar la soberanía costarricense sobre la Isla del Coco, de la cual tomó posesión formal el gobierno de Costa Rica en 1869 , de ahí el envío de una autoridad civil y un resguardo militar, además de la apertura de una comunicación marítima por medio de buques mercantes entre el puerto de Puntarenas y la Bahía Wafer (Arias, 1993). Esta reafirmación territorial se desarrolla en un contexto en el que el país mantiene disputas limítrofes con Colombia y Nicaragua, en los que se hicieron evidentes el interés de Costa Rica por el potencial petrolero en el Valle de Talamanca y los derechos de navegación de embarcaciones costarricenses en las aguas del río San Juan frente al proyecto de construcción de un canal interoceánico (Díaz, 2013; Díaz \& Guerrero, 2018). Incluso las autoridades civiles costarricenses reconocieron la labor de los misioneros enviados por la Diócesis de San José de Costa Rica en los territorios fronterizos con el fin de reafirmar su soberanía sobre ellos (Sandí, 2011). 
Desde el punto de vista histórico-científico, esta expedición proporcionó conocimientos sobre la fauna, la flora y las condiciones geográficas del territorio insular, se analizó la viabilidad de su colonización en una época en que se produjo el fracaso del proyecto de establecer una colonia impulsado por el empresario alemán August Gissler (1857-1935), debido a la distancia que separaba la isla del continente. El patrocinio estatal para estos viajes de estudio científico se refleja en el surgimiento de topónimos vinculados a figuras políticas de la época como los cerros Iglesias - punto culminante de la isla - y Jesús Jiménez, la isla Manuelita y las puntas Montealegre y Rodríguez (Arias, 1993; Díaz \& Alfaro, 2013). De esta forma, la Isla del Coco se constituyó en una prolongación del territorio de la República de Costa Rica en el Océano Pacífico oriental y debido a la difusión de leyendas que la asociaban con el ocultamiento de tesoros por parte de piratas durante el período colonial - desacreditadas por Biolley en su artículo - y el reconocimiento de su potencial económico y posteriormente de su biodiversidad, se fue incorporando paulatinamente como un elemento de la geografía en la identidad nacional costarricense (Díaz, Alfaro \& Ugalde, 2012).

En la primera mitad del siglo XX, con la apertura del Canal de Panamá (1914), se pensó que dicha isla sería un punto de interés para el avituallamiento de las embarcaciones que surcaran las rutas comerciales del Pacífico oriental e incluso, se consideró la posibilidad de establecer una base militar en la década de 1930, especialmente en función de los intereses geopolíticos de los Estados Unidos en la zona, sin embargo, esta idea se disipó una vez que el gobierno estadounidense decidió instalar una base militar en el archipiélago de las Galápagos y se mantuvo activa durante la Segunda Guerra Mundial (1939-1945) (Sylva, 1992; Arias, 1993; Segura, 1996). La expedición científica organizada por el IFGN en 1902, se enmarca dentro del "régimen de cientificidad" propuesto por Viales \& Clare (2009), debido al apoyo proporcionado por el Estado costarricense y la comunidad científica radicada en el país en esa época. De los datos recopilados durante las observaciones hechas in situ, fue posible elaborar un artículo como el de Biolley para el estudio de los moluscos de la Isla del Coco y fue la base para futuras investigaciones como las de Monge-Nájera (1997) y Barrientos (2003).

El intercambio de información científica realizada por Biolley con científicos nacionales (Alfaro y Tristán) y extranjeros (Ancey, Dall $\mathrm{y}$ von Martens) evidencia su pertenencia a una serie de redes de investigación científica. Los estudios de las redes sociales en las que estuvo vinculada la comunidad científica en Costa Rica, correspondientes al período entre finales del siglo XIX e inicios del siglo XX, se reducen básicamente a trabajos que plantean su análisis desde un punto de vista institucional como el de Solano (2007) sobre la Oficina de Estadística, la tesis de Villalobos (2009) respecto a la Sociedad Nacional de Agricultura y el artículo de Díaz \& Calvo (2018) relacionado con el IFGN. Sin embargo, no se han estudiado todavía las redes conformadas por los actores sociales individuales de este período, especialmente a partir del intercambio de conocimientos científicos con investigadores ubicados en el exterior.

Los datos científicos intercambiados por Biolley con investigadores nacionales y extranjeros constituyen un muestra de lo que Solano (1999) denomina "modelo bidireccional" y Azuela \& Lertora (2007) "modelo de red", en el que la transmisión del conocimiento científico se da mediante un intercambio de datos en una doble vía, a través de nodos conformados por actores sociales, individuales y colectivos, en el que cada uno de esos actores puede convertirse en transmisor o receptor de ideas científicas, por lo que cada nodo va transformando esas ideas mediante de un continuo proceso de retroalimentación.

La exploración geográfica del territorio costarricense bajo una perspectiva científica y sistematizada, se desarrolló entre la década de 1890 y la de 1900 , como parte de un proyecto de investigación impulsado por el IFGN que tenía como fin la apropiación del territorio nacional, en particular de las zonas periféricas 
y extracontinentales, en particular de la Isla del Coco, en una época de pugnas limítrofes con los países vecinos. Gracias a la reafirmación de la soberanía costarricense sobre este territorio insular, el país contaba con una isla oceánica que en el futuro sería fundamental para la extensión de sus límites marítimos en el Océano Pacífico oriental.

La exploración de la Isla del Coco permitió un reconocimiento pormenorizado de su topografía y su biodiversidad, con el objetivo de evaluar las posibilidades de su colonización, tras fracasar el proyecto de centro penal y la fallida experiencia de la colonia fundada por Gissler al finalizar XIX. No obstante, su distancia respecto al territorio continental costarricense y su irregular topografía, tanto costera como en su interior, determinaron que el establecimiento de poblaciones humanas permanentes estaba destinada al fracaso, pese al interés que despertaba la futura apertura del Canal de Panamá, que no transformó la isla en un punto de avituallamiento para las embarcaciones que hacían uso de la ruta interoceánica.

No obstante, estas expediciones permitieron el acopio de gran cantidad de datos científicos gracias al inventario de sus recursos naturales, que permitieron el reconocimiento de su flora y su fauna, así como la relación existente con la biodiversidad de las vecinas Galápagos y la costa pacífica centroamericana.

La malacología fue uno de los campos de las ciencias biológicas que más se beneficiaron con estas exploraciones, debido a la recopilación de datos sobre las distintas especies de moluscos que habitaban las costas, los cursos fluviales y la superficie de la Isla del Coco, por lo que Biolley elaboró un inventario de moluscos, que consideró el más completo que se había elaborado hasta ese momento y describió, en forma pormenorizada a la mayor parte de esas especies, lo que permitió identificar sus características, su hábitat y la zona del planeta donde se concentraban principalmente sus poblaciones.

Esta información fue confrontada con los datos procedentes de las publicaciones existentes y mediante el intercambio de criterios con célebres malacólogos de ese tiempo, bajo un modelo de red que permitió la transmisión y retroalimentación de los datos obtenidos, lo que evidencia también la presencia de redes sociales integradas por investigadores residentes en varios países, por lo que este artículo, visto desde el enfoque de la Historia Social de la Ciencia, constituye una contribución al estudio de las redes sociales científicas y en la difusión de los datos que fueron recopilados bajo el denominado "régimen de cientificidad" en Costa Rica.

Declaración de ética: el autor declara que está de acuerdo con esta publicación; que no existe conflicto de interés de ningún tipo; y que ha cumplido con todos los requisitos y procedimientos éticos y legales pertinentes. Todas las fuentes de financiamiento se detallan plena y claramente en la sección de agradecimientos. El respectivo documento legal firmado se encuentra en los archivos de la revista.

\section{AGRADECIMIENTOS}

El autor agradece el apoyo institucional recibido a través del Proyecto "Diccionario histórico-toponímico de la isla del Coco" (VI-805-A9-742) y del Programa de Estudios Sociales de la Ciencia, la Técnica y el Medio Ambiente (PESCTMA, 805-A4-906) del Centro de Investigaciones Geofísicas (CIGEFI) de la Universidad de Costa Rica y a Luis Ricardo Romero Valverde por su aporte en la búsqueda de fuentes para la producción de este artículo $\mathrm{y}$ al señor Michel Montoya por facilitar un ejemplar de la versión original en francés del texto de Biolley.

\section{RESUMEN}

Introducción: El artículo "Moluscos de la Isla del Coco" publicado en la Revista del Colegio Superior de Señoritas (1935) es la traducción al español de una de las últimas obras de Paul Biolley Matthey (1862-1908), naturalista suizo radicado en Costa Rica, producto de una investigación malacológica efectuada en el marco de una expedición científica organizada por el Instituto FísicoGeográfico Nacional de Costa Rica (IFGN) en 1902, en 
una época en que el país reivindicaba su soberanía tanto en este territorio como en las zonas periféricas fronterizas. Objetivo: Analizar los aportes del artículo "Moluscos de la Isla del Coco" desde el enfoque de la Historia Social de la Ciencia. Métodos: La mayor parte de las investigaciones que han aplicado este enfoque en Costa Rica analizan procesos de institucionalización de disciplinas científicas. La metodología empleada en estos trabajos fue de gran utilidad para el análisis del artículo de Biolley, junto con la información de datos históricos proporcionados por otros textos sobre la Isla del Coco y el desarrollo de la investigación científica en Costa Rica desde una perspectiva temporal. Además, se hicieron tablas que sintetizan la clasificación de las especies y géneros estudiados por Biolley en la Isla del Coco para identificar su procedencia y el hábitat en el que viven. Resultados: Este estudio de Biolley constituye un texto de interés desde el punto de vista de la Historia Social de la Ciencia, debido a las descripciones que ofrece de las condiciones físicas del territorio de la Isla del Coco, de las diferentes especies de moluscos localizadas en ellas y al intercambio de información con científicos nacionales y extranjeros que evidencian la participación de Biolley en redes científicas. Conclusiones: Al analizar la información proporcionada por el artículo con los trabajos basados en el enfoque de la Historia Social de la Ciencia, se llegó a la conclusión que la recopilación de los datos de flora y de fauna permitió tener un mayor conocimiento de los recursos naturales de los territorios explorados, con el fin de colonizarlos e integrarlos a la dinámica económica del Valle Central de Costa Rica, sin embargo, los intentos para efectuar una colonización efectiva de la Isla del Coco fracasaron dada la distancia entre esta y el continente, además de las condiciones topográficas que impiden el establecimiento permanente de poblaciones humanas y sus actividades productivas.

Palabras claves: Costa Rica, Isla del Coco, Historia, Geografía, Ciencia, Moluscos.

\section{REFERENCIAS}

Arias, R. (1993). La Isla del Coco: perspectiva histórica y análisis de una leyenda. (Tesis de Licenciatura en Historia). Universidad de Costa Rica, San Pedro, Costa Rica. 200 p.

Azuela, L. F. \& Lértora, C. A. (2007). Introducción. En C. A. Lértora (Coord.), Geonaturalia. Geografia e Historia Natural: hacia una historia comparada. Estudio a través de Argentina, México, Costa Rica y Paraguay (pp. 9-23). Buenos Aires, Argentina: Ediciones FEPAI.

Barrientos, Z. (2003). Lista de especies de moluscos terrestres (Archaeogastropoda, Mesogastropoda, Archaeopulmonata, Stylommatophora, Soleolifera) informadas para Costa Rica. Revista de Biología Tropical, 51(3), 293-304
Biolley, P. (1935). Moluscos de las Isla del Coco. Revista del Colegio de Señoritas (San José) 2(6), 2-18 (Original en francés: Biolley, P. 1907. Mollusques de l'Isla del Coco. Museo Nacional de Costa Rica. Tipografía Nacional, San José, Costa Rica. 30 p.

Coronado, G. (1997). La actividad científica en Costa Rica: bosquejo de su evolución. En Á. Zamora (Comp.), El otro laberinto (tecnología, filosofia, historia) (pp. 257-276). Cartago, Costa Rica: Editorial Tecnológica de Costa Rica.

Cortés, J. (2008). Historia de la investigación marina de la Isla del Coco, Costa Rica. Revista de Biología Tropical, 56(Suplemento 2), 1-18.

Díaz, R. E. (2003). El proceso de institucionalización de la meteorología en Costa Rica (1887-1949). (Tesis de Licenciatura en Historia). Universidad de Costa Rica, San Pedro, Costa Rica. 435 p.

Díaz, R. E. (2007). Exploraciones geográficas e historia natural en Costa Rica: el Instituto Físico-Geográfico Nacional (1889-1903). En C. A. Lértora (Coord.). Geonaturalia. Geografia e Historia Natural: hacia una historia comparada. Estudio a través de Argentina, México, Costa Rica y Paraguay (pp. 205-232). Buenos Aires, Argentina: Ediciones FEPAI.

Díaz, R. E. (2009). Estado, comunidades científicas y exploraciones geográficas en Costa Rica: los proyectos cartográficos del Instituto Físico-Geográfico Nacional (1889-1903). En R. Viales, J. A. Amador \& F. J. Solano (Eds.). Concepciones y representaciones de la naturaleza y la ciencia en América Latina (pp. 211-219). San José, Costa Rica: Universidad de Costa Rica, Vicerrectoría de Investigación.

Díaz, R. E., Alfaro, E. J., \& Ugalde, A. (2012). Diccionario histórico-toponímico de la Isla del Coco, Costa Rica. Revista de Biología Tropical, 60(Suplemento 3), 1-14.

Díaz, R. E., \& Alfaro, E. J. (2013). Diccionario de la toponimia de la Isla del Coco. San José, Costa Rica: Editorial Nuevas Perspectivas.

Díaz, R. E., \& Calvo-Solano, Ó. D. (2018). Instituciones científicas y redes sociales en Costa Rica: El Instituto Físico-Geográfico Nacional (1889-1943). En C. A. Lértora (Coord.), La Reforma Universitaria de 1918 y la ciencia argentina: XIX Jornadas de Historia de la Ciencia: Actas (pp. 79-105). Buenos Aires, Argentina: Ediciones FEPAI.

Díaz, R. E., \& Guerrero, C. (2018). Los orígenes de la exploración petrolera y sus repercusiones ambientales en Costa Rica y Colombia: Un análisis comparativo (1890-1950). En C. García \& C. A. Lértora (Coords.), Los estudios ambientales en Latinoamérica: logros, tendencias y prospectivas: Proyecto Ecoepisteme (pp. 83-107). Buenos Aires, Argentina: Ediciones FEPAI. 
Díaz, R. E., \& Solano, F. J. (2008). Costa Rica: Desarrollo científico. Una mirada en su historia natural a través de Paul Biolley Matthey (1886-1908). En C. A. Lértora (Coord.), Geonaturalia. Geografia e Historia Natural: hacia una historia comparada. Estudio a través de Argentina, México, Costa Rica y Paraguay (pp. 209-238). Buenos Aires, Argentina: Ediciones FEPAI.

Goebel, A. (2009). Naturaleza, mercado y "progreso": las justificaciones de la reorganización productiva de la naturaleza en los exploradores extranjeros en la Costa Rica decimonónica (1850-1905). En R. Viales, J. A. Amador y F. J. Solano (Eds.), Concepciones y representaciones de la naturaleza y la ciencia en América Latina (pp. 111-121). San José, Costa Rica: Universidad de Costa Rica, Vicerrectoría de Investigación.

Monge-Nájera, J. (1997). Moluscos de importancia agrícola y sanitaria en el trópico: la experiencia costarricense / Molluscs of economic and sanitary importance in the Tropics: the Costa Rican experience. San José, Costa Rica: Editorial de la Universidad de Costa Rica.

Quesada, J. R. (2001). Historia de la historiografía costarricense, 1821-1940. San José, Costa Rica: Editorial de la Universidad de Costa Rica.

Saldaña, J. J. (1996). Teatro científico americano. Geografía y cultura en la historiografía latinoamericana de la Ciencia. En J. J Saldaña (Comp.). La historia social de las ciencias en América Latina (pp. 7-41). Ciudad de México, México: Porrúa.

Sandí, J. A. (2011). La participación de la Iglesia Católica en el control del espacio en medio de la creación de un país llamado Costa Rica, 1850-1920. Revista de Historia (Costa Rica), 63-64, 53-99.

Segura, J. (1996). Costa Rica y su contexto internacional. En J. R. Quesada (Ed.), Costa Rica contemporánea. Raices del Estado de la Nación (pp. 309-355). San José, Costa Rica: Editorial de la Universidad de Costa Rica.
Solano, F. J. (1999). El proceso de institucionalización de la meteorología en Costa Rica en el siglo XIX. (Tesis de Licenciatura en Historia). Universidad de Costa Rica, San Pedro, Costa Rica. 240 p.

Solano, F. J. (2007). El clima, la Historia Natural y las instituciones estatales en Costa Rica: el caso de la Oficina de Estadística (1860-1888). En C. A. Lértora (Coord.), Geografia e historia: hacia una historia comparada. Estudio a través de Argentina, México, Costa Rica y Paraguay (pp. 151-181). Buenos Aires, Argentina: Ediciones FEPAI.

Solano, F. J., Díaz, R. E., \& Amador, J. A. (2010). Evolución de las ideas meteorológicas y el impacto del clima en la sociedad costarricense (1502-1860). San José, Costa Rica: Ediciones Sanabria, CIGEFI CIHAC, Universidad de Costa Rica.

Solano, F. J., Díaz, R. E, \& Amador, J. A. (2013). La institucionalización de la meteorología en Costa Rica (1860-1910). San José, Costa Rica: Editorial Nuevas Perspectivas.

Sylva, P. (1992). Las islas Galápagos en la historia del Ecuador. En Nueva historia del Ecuador, 12, Ensayos generales I: Espacio, población, región (pp. 253303). Quito, Ecuador: Corporación Editora Nacional - Grijalbo Ecuatoriana.

Viales, R., \& Clare, P. (2009). El Estado, lo transnacional y la construcción de comunidades científicas en la Costa Rica liberal (1870-1930). La construcción de un "régimen de cientificidad". En R. Viales, J. A. Amador, \& F. J. Solano (Eds), Concepciones y representaciones de la naturaleza y la ciencia en América Latina (pp. 97-109). San José, Costa Rica: Universidad de Costa Rica, Vicerrectoría de Investigación.

Villalobos, G. (2009). "El progreso redentor". La Sociedad Nacional de Agricultura, el Estado liberal y la modernización agropecuaria en Costa Rica, 1897 1914. (Tesis de Maestría en Historia). Universidad de Costa Rica. San Pedro, Costa Rica. 326 p. 